\title{
ATTITUDE ESTIMATION FOR LARGE FIELD-OF-VIEW SENSORS
}

\author{
Yang Cheng \\ John L. Crassidis
}

University at Buffalo, State University of New York Department of Mechanical \& Aerospace Engineering Amherst, NY 14260-4400

\section{F. Landis Markley}

NASA Goddard Space Flight Center Guidance, Navigation and Control Systems Eng. Branch Greenbelt, MD 20771

\section{The Malcolm D. Shuster \\ Astronautics Symposium}

Grand Island, New York

13-15 June 2005

AAS Publications Office, P.O. Box 28130, San Diego, CA 92198 
AAS 05-462

\title{
ATTITUDE ESTIMATION FOR LARGE FIELD-OF-VIEW SENSORS
}

\author{
Yang Cheng*, John L. Crassidis ${ }^{\dagger}$ and F. Landis Markley
}

The QUEST measurement noise model for unit vector observations has been widely used in spacecraft attitude estimation for more than twenty years. It was derived under the approximation that the noise lies in the tangent plane of the respective unit vector and is axially symmetrically distributed about the vector. For large field-of-view sensors, however, this approximation may be poor, especially when the measurement falls near the edge of the field of view. In this paper a new measurement noise model is derived based on a realistic noise distribution in the focal-plane of a large field-of-view sensor, which shows significant differences from the QUEST model for unit vector observations far away from the sensor boresight. An extended Kalman filter for attitude estimation is then designed with the new measurement noise model. Simulation results show that with the new measurement model the extended Kalman filter achieves better estimation performance using large field-of-view sensor observations.

\section{INTRODUCTION}

\begin{abstract}
TTITUDE determination is the identification of a proper orthogonal rotation (attitude) Amatrix that maps sensed vectors from a reference frame into the sensor frame. If all the measured and reference vectors are error free, then the rotation matrix is the same for all sets of observations. However, if measurement errors exist, then a least-squares type approach must be used to determine the attitude. Several attitude sensors exist, including: three-axis magnetometers, sun sensors, Earth-horizon sensors, global positioning system (GPS) sensors and star trackers. Reference [1] provides detailed descriptions of each of these sensors. The specific choice for the complement of onboard attitude sensor hardware is mostly driven by the individual requirements of the spacecraft mission. For example, for low accuracy requirements, such as a few degrees, a three-axis magnetometer can be used solely to determine three-axis attitude coupled with gyroscopes or a dynamic model [2]. The Solar, Anomalous, and Magnetospheric Particle Explorer (SAMPEX) is an example of a highly successful mission that employs only a three-axis magnetometer and a coarse $(0.25$ degree) sun sensor coupled with a dynamic model [3].
\end{abstract}

\footnotetext{
*Postdoctoral Research Fellow, Department of Mechanical \& Aerospace Engineering, University at Buffalo, State University of New York, Amherst, NY 14260-4400. E-mail: yangc0@yahoo.com.

${ }^{\dagger}$ Associate Professor, Department of Mechanical \& Aerospace Engineering, University at Buffalo, State University of New York, Amherst, NY 14260-4400. E-mail: johnc@eng.buffalo.edu.

${ }^{\ddagger}$ Aerospace Engineer, NASA Goddard Space Flight Center, Guidance, Navigation and Control Systems Engineering Branch, Greenbelt, MD 20771. E-mail: Landis.Markley@nasa.gov.
} 
For missions with tight attitude knowledge requirements, the primary means to determine attitude is the star tracker. The technology behind star trackers has changed much over the years. Evolving from gimballed to fixed-head, the latest star trackers now use charge-coupled devices (CCD) for imaging, which offer high accuracy [4]. By using star image centroiding, accuracies of approximately $1 / 10$ the size of a pixel can be achieved. For small field-of-view (FOV) star trackers, this leads to off-boresight attitude knowledge on the order of 10 to 20 arcsec. Star trackers fall into the category of line-of-sight (LOS) sensors since they measure the direction of a celestial body. In particular, the angle of that body is measured from the sensor boresight in two mutually orthogonal planes [5]. With the advent of low-cost CCD arrays and powerful processors, the use of star trackers is more common today.

For LOS sensors the observation equations are given by the well-known collinearity equations [6], which relate image plane coordinates to object plane coordinates through an attitude rotation. For stellar applications the light sources can be treated as infinite distance points, so that the only unknown, once a star is identified, is the attitude matrix. All attitude sensors, including star trackers, contain noise in their measurements however. This noise includes both systematic errors and random errors. Systematic errors are reduced through calibration procedures, which can even be done on-orbit [7]. Random errors are usually treated as zero-mean Gaussian white-noise processes with known covariance. A realistic covariance matrix takes into account an increase in the errors away from the boresight due to radial distortions and contains correlated terms. A frequently-used covariance model for the noise added to the collinearity truth equations is given by Eq. (10) of Ref. [8]. This model is referred to here as the "focal-plane model."

The collinearity equations are usually cast in vector form since the attitude matrix appears linearily in this form. This unit vector form, also called the "LOS measurement model," is the most widely used observation equation in attitude determination [9]. Recent research has also shown that using the unit vector form produces better results in a filter design over the standard collinearity equations form for the observation equations [10]. This is due to lower nonlinearity of the LOS measurement model and boundedness of the LOS measurements. Unfortunately, the measurement noise is also transformed when the collinearity equations are converted into the unit vector form. A simple covariance model that is valid for small FOVs has been developed by Shuster and Oh [9], called the "QUEST measurement model," which is a singular matrix that arises for the unit-normalization of the observations. The beauty of this model is in its simplicity, which is evident in its use in the extended Kalman filter (EKF). In particular, Shuster [8] has shown that the singular covariance matrix can effectively be replaced with a nonsingular isotropic matrix, thereby providing practical use in the EKF.

In this paper the QUEST measurement model is replaced with a general model that is valid for large FOVs. New sensors are evolving that incorporate wider FOVs, which may lead to degraded performance when using the QUEST measurement model. One such sensor is the vision-based navigation (VISNAV) system [11], which comprises an optical sensor of a new kind combined with specific light sources (beacons) in order to achieve a selective or "intelligent" vision. The sensor is made up of a Position Sensing Diode (PSD) placed in the focal plane of a wide angle lens, which yields a 100 degree FOV. When the rectangular silicon area of the PSD is illuminated by energy from a beacon focused by the 


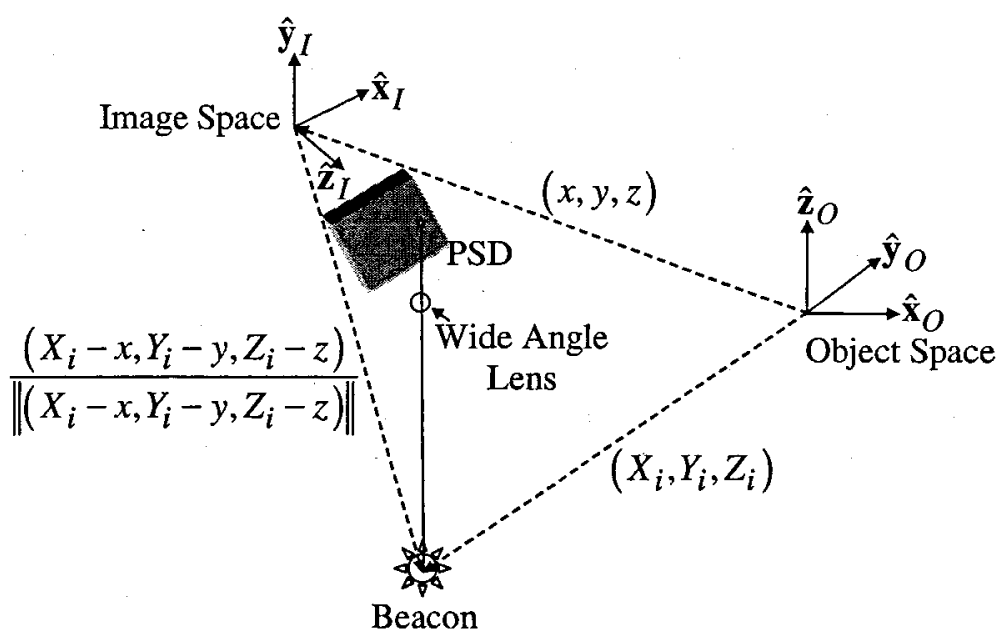

Figure 1 Vision Based Navigation System

lens, it generates electrical currents in four directions that can be processed with appropriate electronic equipment to estimate the energy centroid of the image. The new measurement model is derived using a first-order Taylor series expansion approach of the observation noise model. This makes the assumption that the noise is "small" compared to the signal. It will be shown that this model can produce more accurate results than the QUEST measurement model for the VISNAV sensor. Unfortunately, as is the case with the QUEST measurement model, the new covariance is also singular, which causes a problem in the computation of the Kalman gain. To overcome this problem, two solutions are presented. The first is based on a matrix decomposition of the new covariance and the second is based on a rank-one update.

The organization of this paper proceeds as follows. First, the collinearity equations are summarized, followed by the introduction of the focal-plane covariance model. Then, the QUEST measurement model is reviewed. Next, a new covariance model is derived that is valid for large FOVs. Its implementation in an EKF design is then shown by using a measurement transformation approach as well as a rank-one update approach. Finally, simulation results are shown that compare EKF results with the QUEST measurement model versus the new measurement model using VISNAV sensor observations.

\section{OVERVIEW}

In this section the collinearity equations are summarized for close range photogrammetry applications using the VISNAV sensor. A covariance model for the focal-plane equations is also summarized and the QUEST measurement model is shown.

\section{Collinearity Equations}

Photogrammetry is the technique of measuring objects (2D or 3D) from photographic images or LOS measurements. Photogrammetry can generally be divided into two categories: far range photogrammetry with camera distance settings to infinity (commonly used in star cameras), and close range photogrammetry with camera distance settings to finite 
values. In general close range photogrammetry can be used to determine both the position and attitude of an object, while far range photogrammetry can only be used to determine attitude. The VISNAV system comprises an optical sensor of a new kind combined with specific light sources (beacons), which can be used for close range photogrammetry-type applications. The relationship between the position/attitude and the observations used in photogrammetry involves a set of collinearity equations, which are reviewed in this section. Figure 1 shows a schematic of the typical quantities involved in basic photogrammetry from LOS measurements, derived from light beacons in this case. If we choose the $z$-axis of the sensor coordinate system to be directed outward along the boresight, then given object space and image space coordinate frames (see Figure 1), the ideal object to image space projective transformation (noiseless) can be written as follows [6]:

$$
\begin{aligned}
\alpha_{i} & =-f \frac{A_{11}\left(X_{i}-x\right)+A_{12}\left(Y_{i}-y\right)+A_{13}\left(Z_{i}-z\right)}{A_{31}\left(X_{i}-x\right)+A_{32}\left(Y_{i}-y\right)+A_{33}\left(Z_{i}-z\right)}, \quad i=1,2, \ldots, N \\
\beta_{i} & =-f \frac{A_{21}\left(X_{i}-x\right)+A_{22}\left(Y_{i}-y\right)+A_{23}\left(Z_{i}-z\right)}{A_{31}\left(X_{i}-x\right)+A_{32}\left(Y_{i}-y\right)+A_{33}\left(Z_{i}-z\right)}, \quad i=1,2, \ldots, N
\end{aligned}
$$

where $N$ is the total number of observations, $\left(\alpha_{i}, \beta_{i}\right)$ are the image space observations for the $i^{\text {th }} \operatorname{LOS},\left(X_{i}, Y_{i}, Z_{i}\right)$ are the known object space locations of the $i^{\text {th }}$ beacon, $(x, y, z)$ are the unknown object space location of the sensor, $f$ is the known focal length, and $A_{j k}$ are the unknown coefficients of the attitude matrix, $A$. In general the observations can be given by $\alpha_{i} / f$ and $\beta_{i} / f$, so we can assume $f=1$ without loss in generality, which is done for the remainder of this paper. The goal of the inverse problem is given observations $\left(\alpha_{i}, \beta_{i}\right)$ and object space locations $\left(X_{i}, Y_{i}, Z_{i}\right)$, for $i=1,2, \ldots, N$, determine the attitude $(A)$ and position $(x, y, z)$. Note that if the beacons are "infinitely away" then Eq. (1) reduces down to the standard stellar collinearity equations.

Denoting $\alpha_{i}$ and $\beta_{i}$ by the $2 \times 1$ vector $\gamma_{i} \equiv\left[\alpha_{i} \beta_{i}\right]^{T}$, then the measurement model follows

$$
\tilde{\gamma}_{i}=\gamma_{i}+\mathbf{w}_{i}
$$

where a frequently used covariance for $\mathrm{w}_{i}$ with $f=1$ is given by [8]

$$
R_{i}^{\mathrm{FOCAL}}=\frac{\sigma^{2}}{1+d\left(\alpha_{i}^{2}+\beta_{i}^{2}\right)}\left[\begin{array}{cc}
\left(1+d \alpha_{i}^{2}\right)^{2} & \left(d \alpha_{i} \beta_{i}\right)^{2} \\
\left(d \alpha_{i} \beta_{i}\right)^{2} & \left(1+d \beta_{i}^{2}\right)^{2}
\end{array}\right]
$$

where $d$ is on the order of one and $\sigma$ is assumed to be known. Note that as $\alpha_{i}$ or $\beta_{i}$ increase then the individual components of $R_{i}^{\mathrm{FOCAL}}$ increase, which realistically shows that the errors increase as the observation moves away from the boresight. Also, as stated in Ref. [8], the covariance model is a function of the true variables $\alpha_{i}$ and $\beta_{i}$, which are never available in practice. However, using the measurements themselves or estimated quantities in the EKF leads to only second-order error effects.

\section{Unit Vector Form}

The observation can be reconstructed in unit vector form as

$$
\mathbf{b}_{i}=A \mathbf{r}_{i}, \quad i=1,2, \ldots, N
$$


where

$$
\begin{gathered}
\mathbf{b}_{i} \equiv \frac{1}{\sqrt{1+\alpha_{i}^{2}+\beta_{i}^{2}}}\left[\begin{array}{c}
-\alpha_{i} \\
-\beta_{i} \\
1
\end{array}\right] \\
\mathbf{r}_{i} \equiv \frac{1}{\sqrt{\left(X_{i}-x\right)^{2}+\left(Y_{i}-y\right)^{2}+\left(Z_{i}-z\right)^{2}}}\left[\begin{array}{c}
X_{i}-x \\
Y_{i}-y \\
Z_{i}-z
\end{array}\right]
\end{gathered}
$$

When measurement noise is present, Shuster and $\mathrm{Oh}$ [9] have shown that nearly all the probability of the errors is concentrated on a very small area about the direction of $A \mathbf{r}_{i}$, so the sphere containing that point can be approximated by a tangent plane, characterized by

$$
\tilde{\mathbf{b}}_{i}=A \mathbf{r}_{i}+\boldsymbol{v}_{i}, \quad v_{i}^{T} A \mathbf{r}_{i}=0
$$

where $\tilde{b}_{i}$ denotes the $i^{\text {th }}$ measurement and the sensor error $v_{i}$ is approximately Gaussian, which satisfies

$$
\begin{gathered}
E\left\{v_{i}\right\}=0 \\
R_{i}^{\text {QUEST }} \equiv E\left\{v_{i} v_{i}^{T}\right\}=\sigma^{2}\left[I_{3 \times 3}-\left(A \mathbf{r}_{i}\right)\left(A r_{i}\right)^{T}\right]
\end{gathered}
$$

where $E\{\}$ denotes expectation and $I_{3 \times 3}$ denotes a $3 \times 3$ identity matrix. Equation (7b) is known as the QUEST measurement model. Shuster has shown that for $\alpha_{i}^{2}+\beta_{i}^{2} \ll 1$, the QUEST measurement model agrees well with the inferred model for the real sensor given by Eq. (3). Note that Eq. (7b) is also a function of the unknown truth quantities. However, the advantage of using the QUEST measurement model is that the measurement covariance in the EKF formulation can effectively be replaced by a nonsingular matrix, given by $\sigma_{i}^{2} I_{3 \times 3}$ (see Ref. [8] for more details).

\section{New Model}

To derive a covariance for the actual unit vector measurement, the true values for $\alpha_{i}$ and $\beta_{i}$ must be replaced with the measured ones in Eq. (5). Performing this replacement does not explicitly yield the form given by Eq. (6) because the actual model cannot separate $A r_{i}$ from the noise. Hence, the actual noise model contains nonlinear terms coupled with non-Gaussian components. In order to derive a covariance, the new measurement model is based on a first-order Taylor series expansion of the unit vector model in Eq. (6). Note that this approach does not make the small FOV assumption, but rather it makes the assumption that the measurement noise is "small" compared to the signal, which is valid for every star tracker and for the VISNAV sensor as well. The Jacobian of Eq. (5a) is given by

$$
J_{i} \equiv \frac{\partial \mathbf{b}_{i}}{\partial \gamma_{i}}=\frac{1}{\sqrt{1+\alpha_{i}^{2}+\beta_{i}^{2}}}\left[\begin{array}{cc}
-1 & 0 \\
0 & -1 \\
0 & 0
\end{array}\right]-\frac{1}{1+\alpha_{i}^{2}+\beta_{i}^{2}} \mathbf{b}_{i}\left[\begin{array}{ll}
\alpha_{i} & \beta_{i}
\end{array}\right]
$$

The new covariance is now given by

$$
R_{i}^{\mathrm{NEW}}=J_{i} R_{i}^{\mathrm{FOCAL}} J_{i}^{T}
$$

Clearly, $R_{i}^{\mathrm{NEW}}$ is a singular matrix, just as Eq. (7b). It will be shown that the eigenvector associated with the zero eigenvalue of $R_{i}^{\mathrm{NEW}}$ is $A \mathbf{r}_{i}$, which is exactly the same eigenvector 
associated with the zero eigenvalue of $R_{i}^{\text {QUEST }}$. Since $R_{i}^{\text {QUEST }}$ has two repeated eigenvalues, $\sigma^{2}$, then the associated eigenvectors, which are always in the plane perpendicular to $A \mathrm{r}_{i}$, are not unique. Therefore, without loss in generality it can be assumed that $R_{i}^{\text {QUEST }}$ has the same eigenvectors as $R_{i}^{\mathrm{NEW}}$. Thus, the only differences between these two covariances are their nonzero eigenvalues. The covariances are identical, i.e. $R_{i}^{\mathrm{NEW}}=R_{i}^{\mathrm{QUEST}}$, when $R_{i}^{\text {FOCAL }}$ is given by

$$
R_{i}^{\mathrm{FOCAL}}=\sigma^{2}\left(1+\alpha_{i}^{2}+\beta_{i}^{2}\right)\left[\begin{array}{cc}
1+\alpha_{i}^{2} & \alpha_{i} \beta_{i} \\
\alpha_{i} \beta_{i} & 1+\beta_{i}^{2}
\end{array}\right]
$$

which agrees with the corrected result shown in Ref. [12]. This shows that in order to recover $R_{i}^{\text {QUEST }}$, the errors also should not be constant over the FOV. Note the differences between Eq. (3) and Eq. (10) though. However, for small FOVs the condition $\alpha_{i}^{2}+\beta_{i}^{2} \ll 1$ is valid, so Eq. (10) agrees well with Eq. (3) in this case [8].

\section{EXTENDED KALMAN FILTER IMPLEMENTATION}

In this section the new covariance is implemented in the EKF. Two approaches to overcome the singularity issue in the EKF are presented. The first is based on an eigenvalue/eigenvector decomposition of $R_{i}^{\mathrm{NEW}}$ and the second is based on a rank-one update of the covariance matrix. Only attitude estimation is considered here. The VISNAV sensor is capable of determining position as well, but this is an easy extension of the attitude estimation problem by simply augmenting the state vector. The EKF equations follow the multiplicative quaternion approach of Ref. [13], which includes the three-component attitude error vector and gyro-bias errors. Unfortunately, straightforward implementation of the EKF with the new model is not possible. The Kalman gain for a single observation, written in terms of using the true values for now, is given by [14]

$$
K=P^{-} H_{i}^{T}\left[H_{i} P^{-} H_{i}^{T}+R_{i}^{\mathrm{NEW}}\right]^{-1}
$$

where $P^{-}$is the propagated $6 \times 6$ covariance matrix and $H_{i}$ is given by [13]

$$
H_{i}=\left[\begin{array}{ll}
{\left[A \mathrm{r}_{i} \times\right]} & 0_{3 \times 3}
\end{array}\right]
$$

where $\left[A \mathbf{r}_{i} \times\right]$ is the standard cross product matrix (see Ref. [13]) and $0_{3 \times 3}$ is a $3 \times 3$ matrix of zeros. We now investigate the properties of the matrix $Z_{i} \equiv H_{i} P^{-} H_{i}^{T}+R_{i}^{\mathrm{NEW}}$, which is known as the innovation matrix. In the EKF formulation the estimated values will be used to form the matrices $H_{i} P^{-} H_{i}^{T}$ and $R_{i}^{\mathrm{NEW}}$. Hence, for the analysis of the matrix $Z_{i}$ we can set $\mathbf{b}_{i}=A \mathbf{r}_{i}$ without loss in generality for now. From the definition of the cross product matrix it is easy to see that $H_{i}^{T} \mathbf{b}_{i}=\mathbf{0}$. Performing the multiplication $J_{i}^{T} \mathbf{b}_{i}$ also gives $J_{i}^{T} \mathbf{b}_{i}=\mathbf{0}$. Hence, $Z_{i} \mathbf{b}_{i}$ is always zero, which means $Z_{i}$ is always singular. Furthermore, since the rank of $R_{i}^{\mathrm{FOCAL}}$ is two, which means the rank of $R_{i}^{\mathrm{NEW}}$ is also two, then $\mathrm{b}_{i}$ is the eigenvector associated with the zero eigenvalue of $R_{i}^{\mathrm{NEW}}$. The singularity problem always exists no matter how many measurements are used in the EKF. Therefore, the standard EKF cannot be executed.

The specific form for the EKF follows Murrell's version [15], which processes one unit vector observation at a time through a sequential approach. This approach reduces taking 


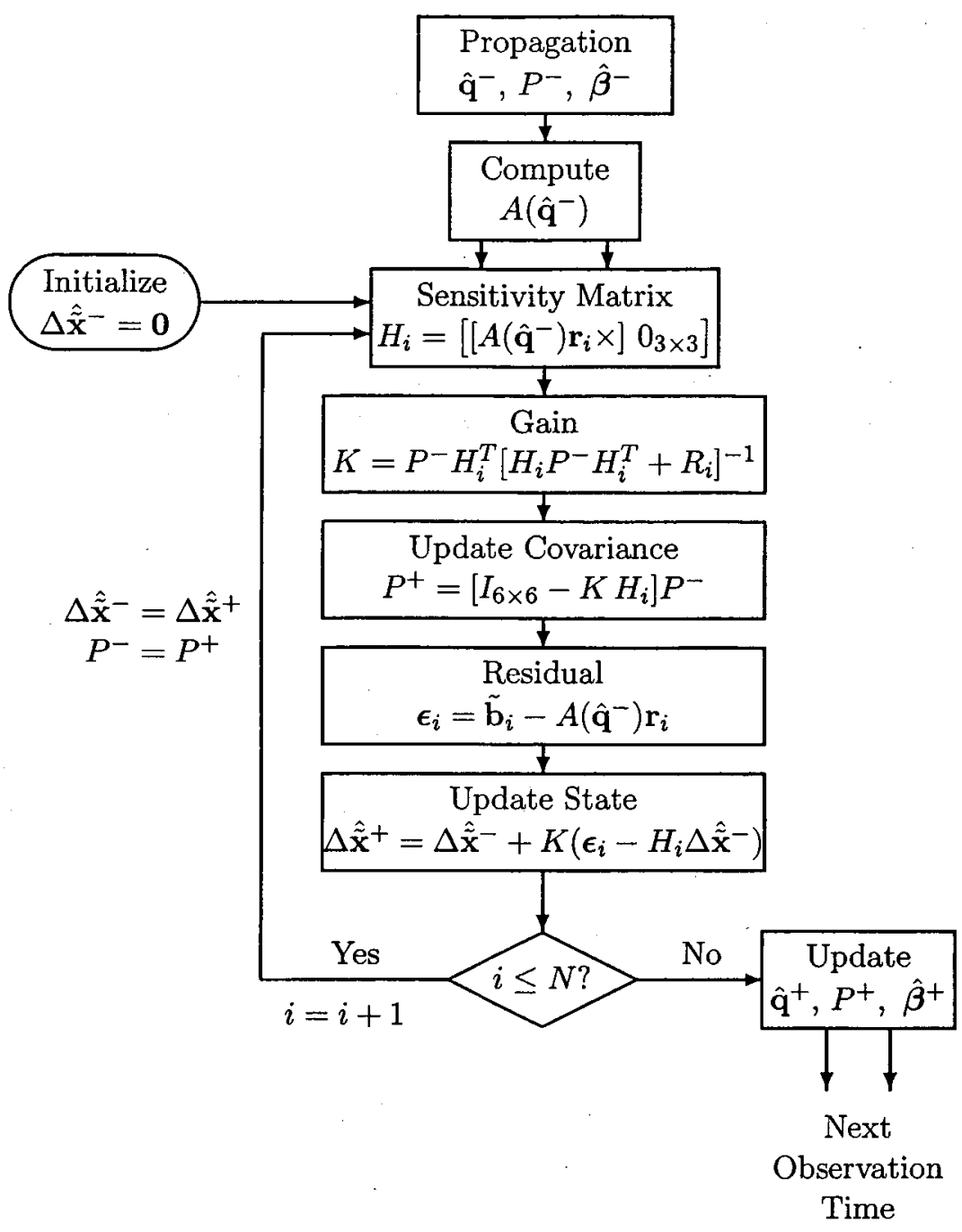

Figure 2 Computationally Efficient Attitude Estimation Algorithm

an inverse of a $3 N \times 3 N$ matrix for the Kalman gain to taking an inverse of a $3 \times 3$ matrix $N$ times, which significantly reduces the computational load. A flow chart of Murrell's version for the update is shown in Figure 2, where $\hat{\boldsymbol{\beta}}^{-}$and $\hat{\boldsymbol{\beta}}^{+}$are the propagated and updated gyrobias estimates, respectively, $P^{+}$is the updated $6 \times 6$ covariance matrix, $\Delta \hat{\tilde{\mathbf{x}}}^{-}$and $\Delta \hat{\tilde{\mathbf{x}}}^{+}$are the propagated and updated state corrections for the attitude and gyro bias, respectively, and $A\left(\hat{\mathrm{q}}^{-}\right)$is the attitude matrix parameterized using the propagated quaternion estimate, $\hat{\mathbf{q}}^{-}$. Note that the gain in this flowchart uses the QUEST measurement model approach, with $R_{i}=\sigma^{2} I_{3 \times 3}$. The first step involves propagating the quaternion, gyro bias and errorcovariance to the current observation time. Then, the attitude matrix is computed. The propagated state vector is now initialized to zero. Next, the error-covariance and state quantities are updated using a single vector observation. This procedure is continued, replacing the propagated error-covariance and state vector with the updated values, until all vector observations are processed. Finally, the updated values are used to propagate the error-covariance and state quantities to the next observation time. 


\section{Decomposition Approach}

The eigenvalue/eigenvector decomposition of $R_{i}^{\mathrm{NEW}}$ can be written in the form given by

$$
\begin{aligned}
R_{i}^{\mathrm{NEW}} & =T_{i} E_{i} T_{i}^{T} \\
& \equiv\left[\begin{array}{lll}
\mathbf{t}_{1} & \mathbf{t}_{2} & \mathbf{t}_{3}
\end{array}\right]_{i}\left[\begin{array}{ccc}
\lambda_{1} & 0 & 0 \\
0 & \lambda_{2} & 0 \\
0 & 0 & 0
\end{array}\right]_{i}\left[\begin{array}{lll}
\mathbf{t}_{1} & \mathbf{t}_{2} & \mathbf{t}_{3}
\end{array}\right]_{i}^{T}
\end{aligned}
$$

where $t_{1}, t_{2}$ and $t_{3}$ are the eigenvectors, and $\lambda_{1}$ and $\lambda_{2}$ are the nonzero eigenvalues. $A$ linear transformation of the measurement residual in Figure 2 is now performed, giving a new residual $\mathrm{d}_{i}$ :

$$
\mathbf{d}_{i} \equiv\left[\begin{array}{l}
\mathbf{e}_{i} \\
g_{i}
\end{array}\right]=T_{i}^{T} \boldsymbol{\epsilon}_{i}
$$

where $\mathbf{e}_{i}$ is a $2 \times 1$ vector made up of the first two components of $\mathbf{d}_{i}$ and $g_{i}$ is the third component of $\mathbf{d}_{i}$. Since $\mathbf{t}_{3}=A\left(\hat{\mathbf{q}}^{-}\right) \mathbf{r}_{i}$, then the transformed sensitivity matrix has the form

$$
T_{i}^{T} H_{i}=\left[\begin{array}{cc}
\mathcal{T}_{i} & 0_{2 \times 3} \\
\mathbf{0}^{T} & \mathbf{0}^{T}
\end{array}\right]
$$

where $\mathcal{T}_{i}$ is a $2 \times 3$ matrix and $0_{2 \times 3}$ is a $2 \times 3$ matrix of zeros. Hence, only the vector $\mathbf{e}_{i}$ is needed to perform the updates. These equations are now given by

$$
\begin{gathered}
\mathcal{K}=P^{-} \mathcal{H}_{i}^{T}\left[\mathcal{H}_{i} P^{-} \mathcal{H}_{i}^{T}+\Lambda_{i}\right]^{-1} \\
P^{+}=\left[I_{6 \times 6}-\mathcal{K} \mathcal{H}_{i}\right] P^{-} \\
\Delta \hat{\tilde{\mathbf{x}}}^{+}=\Delta \tilde{\tilde{\mathbf{x}}}^{-}+\mathcal{K}\left(\mathbf{e}_{i}-\mathcal{H}_{i} \Delta \hat{\tilde{\mathbf{x}}}^{-}\right)
\end{gathered}
$$

where $\mathcal{H}_{i} \equiv\left[\begin{array}{ll}\mathcal{T}_{i} & 0_{2 \times 3}\end{array}\right]$ and $\Lambda_{i}$ is a diagonal matrix made up of the nonzero eigenvalues of $R_{i}^{\mathrm{NEW}}$ :

$$
\Lambda_{i} \equiv\left[\begin{array}{cc}
\lambda_{1} & 0 \\
0 & \lambda_{2}
\end{array}\right]_{i}
$$

Note that the matrix inverse in the Kalman gain reduces from a $3 \times 3$ matrix to a $2 \times 2$ matrix. Also, since $\Lambda_{i}$ is a diagonal matrix, then a sequential process can be used to process each component of $\mathbf{e}_{i}$ one at a time [14]. This reduces taking a $2 \times 2$ matrix inverse down to taking an inverse of a scalar twice, which further reduces the computational load. The quaternion and gyro-bias updates are given by

$$
\begin{gathered}
\Delta \hat{\tilde{\mathbf{x}}}^{+} \equiv\left[\begin{array}{ll}
\boldsymbol{\delta} \hat{\boldsymbol{\alpha}}^{+T} & \Delta \hat{\boldsymbol{\beta}}^{+T}
\end{array}\right]^{T} \\
\hat{\mathbf{q}}^{+}=\hat{\mathbf{q}}^{-}+\frac{1}{2} \Xi\left(\hat{\mathbf{q}}^{-}\right) \boldsymbol{\delta} \hat{\boldsymbol{\alpha}}^{+}, \quad \text { re-normalize quaternion } \\
\hat{\boldsymbol{\beta}}^{+}=\hat{\boldsymbol{\beta}}^{-}+\Delta \hat{\boldsymbol{\beta}}^{+}
\end{gathered}
$$

where $\delta \hat{\alpha}$ is the angle correction, $\Delta \hat{\boldsymbol{\beta}}$ is the bias correction and $\Xi\left(\hat{\mathbf{q}}^{-}\right)$is a $4 \times 3$ matrix given by

$$
\Xi\left(\hat{\mathbf{q}}^{-}\right) \equiv\left[\begin{array}{c}
\hat{q}_{4}^{-} I_{3 \times 3}+\left[\hat{\boldsymbol{\rho}}^{-} \times\right] \\
-\hat{\boldsymbol{\rho}}^{-T}
\end{array}\right]
$$


with $\hat{\mathbf{q}}^{-} \equiv\left[\begin{array}{ll}\hat{\boldsymbol{\rho}}^{-T} & \hat{q}_{4}^{-}\end{array}\right]^{T}$. The propagation equations remain unchanged, which are not shown here for brevity (see Ref. [13] for more details). In order to further enhance the numerical properties of the algorithm, a $U-D$ factorization [14] of the covariance update and propagation is employed. The main advantage of this approach is that the factorization is accomplished without taking square roots, unlike a square-root filter, and the formulation does not add much computational effort over the standard EKF. The new model uses the specific covariance shown in Eq. (3), but it is important to state that any covariance matrix can be used in this formulation. The eigenvalue/eigenvector decomposition can easily be performed using numerical techniques.

\section{Rank-One Update Approach}

An alternative approach to overcome the problem of the EKF due to the singularity of the new measurement covariance matrix is shown. The main idea of the approach is to add an extra term, $c \mathbf{b}_{i} \mathbf{b}_{i}^{T}(c>0)$, to the singular measurement covariance matrix to ensure that the modified measurement covariance matrix is nonsingular. After this modification the standard EKF equations are used, as shown in Figure 2. The EKF with the modified measurement covariance matrix will yield the identical measurement update result with the one employing size-reduced residuals and measurement covariances. This approach is a straightforward extension of Shuster's approach in Ref. [8] to overcome the problem with the singular QUEST measurement covariance matrix (and singular innovation matrix). In Ref. [8] the original QUEST measurement covariance matrix in the standard EKF,$R_{i}^{\text {QUEST }}=\sigma_{i}^{2}\left(I_{3 \times 3}-\mathbf{b}_{i} \mathbf{b}_{i}^{T}\right)$, is replaced by $\mathcal{R}_{i}^{\text {QUEST }}=\sigma_{i}^{2} I_{3 \times 3}$, with the modification to the QUEST measurement covariance given by

$$
\mathcal{R}_{i}^{\mathrm{QUEST}}=R_{i}^{\mathrm{QUEST}}+\sigma^{2} \mathbf{b}_{i} \mathbf{b}_{i}^{T}
$$

Again note that $R_{i}^{\mathrm{QUEST}} \mathrm{b}_{i}=\mathbf{0}$.

For the new measurement model, we propose to modify the singular measurement covariance matrix, $R_{i}^{\mathrm{NEW}}$, in a similar manner:

$$
\mathcal{R}_{i}^{\mathrm{NEW}}=R_{i}^{\mathrm{NEW}}+c_{i} \mathbf{b}_{i} \mathbf{b}_{i}^{T}
$$

with $c_{i}>0$. For any $R_{i}^{\mathrm{NEW}}$ derived from $R_{i}^{\mathrm{FOCAL}}$, it is also true that $R_{i}^{\mathrm{NEW}} \mathbf{b}_{i}=\mathbf{0}$, which is a direct result of $J_{i}^{T} \mathbf{b}_{i}=0$. The inverse of $\mathcal{R}_{i}^{\mathrm{NEW}}$ is then given by

$$
\left(\mathcal{R}_{i}^{\mathrm{NEW}}\right)^{-1}=\left(R_{i}^{\mathrm{NEW}}\right)^{\dagger}+\frac{1}{c_{i}} \mathbf{b}_{i} \mathbf{b}_{i}^{T}
$$

where $\left(R_{i}^{\mathrm{NEW}}\right)^{\dagger}$ may be interpreted as the pseudo-inverse of $R_{i}^{\mathrm{NEW}}$ or simply a convenient notation. Pre-multiplying both sides of Eq. (22) by $H_{i}^{T}$ leads to

$$
H_{i}^{T}\left(\mathcal{R}_{i}^{\mathrm{NEW}}\right)^{-1}=H_{i}^{T}\left(R_{i}^{\mathrm{NEW}}\right)^{\dagger}+H_{i}^{T} \cdot \frac{1}{c_{i}} \mathbf{b}_{i} \mathbf{b}_{i}^{T}=H_{i}^{T}\left(R_{i}^{\mathrm{NEW}}\right)^{\dagger}
$$

Note that because $H_{i}^{T} \mathbf{b}_{i}=\mathbf{0}$, the identity holds regardless of the value of $c_{i}>0$. The limit of $H_{i}^{T}\left(\mathcal{R}_{i}^{\mathrm{NEW}}\right)^{-1}$ as $c_{i}$ approaches zero is $H_{i}^{T}\left(R_{i}^{\mathrm{NEW}}\right)^{\dagger}$ as well. Similarly, for all $c_{i}$ we have

$$
H_{i}^{T}\left(\mathcal{R}_{i}^{\mathrm{NEW}}\right)^{-1} H_{i}=H_{i}^{T}\left(R_{i}^{\mathrm{NEW}}\right)^{\dagger} H_{i}
$$


The inverse of $\mathcal{R}_{i}^{\mathrm{NEW}}$ always exist since the eigenvalues obey [16]

$$
\lambda_{j}\left(\mathcal{R}_{i}^{\mathrm{NEW}}\right)=\lambda_{j}\left(R_{i}^{\mathrm{NEW}}\right)+m_{j} c_{i}, \quad j=1,2,3
$$

with $m_{1}+m_{2}+m_{3}=1$. In fact the eigenvalues and eigenvectors of $\mathcal{R}_{i}^{\mathrm{NEW}}$ are identical to the ones of $R_{i}^{\mathrm{NEW}}$, except that the zero eigenvalue of $R_{i}^{\mathrm{NEW}}$ is replaced with $c_{i}$. Since $c_{i}$ is assumed to never be zero, then the inverse of $\mathcal{R}_{i}^{\mathrm{NEW}}$ always exists.

The measurement update of the covariance matrix and the computation of the Kalman gain matrix with the original and modified measurement covariance matrices may be rewritten as

$$
\begin{aligned}
\left(P^{+}\right)^{-1} & =\left(P^{-}\right)^{-1}+H_{i}^{T}\left(R_{i}^{\mathrm{NEW}}\right)^{\dagger} H_{i} \\
K & =P^{+} H_{i}^{T}\left(R_{i}^{\mathrm{NEW}}\right)^{\dagger}
\end{aligned}
$$

and

$$
\begin{aligned}
\left(P^{+}\right)^{-1} & =\left(P^{-}\right)^{-1}+H_{i}^{T}\left(\mathcal{R}_{i}^{\mathrm{NEW}}\right)^{-1} H_{i} \\
K & =P^{+} H_{i}^{T}\left(\mathcal{R}_{i}^{\mathrm{NEW}}\right)^{-1}
\end{aligned}
$$

From the two sets of equations and Eqs. (23) and (24), we can see that any $\mathcal{R}_{i}^{\mathrm{NEW}}$ will lead to the same $P^{+}$and $K$ and thus to the same measurement update result as $R_{i}^{\mathrm{NEW}}$ does. Therefore, the use of $\mathcal{R}_{i}^{\mathrm{NEW}}$ overcomes the singularity problem, but does not alter the result of the EKF. Finally, we note that

1. There is no limitation of the parameter $c_{i}>0$ in order to guarantee non-singularity of the measurement covariance matrix and the innovation matrix. Physically, $c_{i}$ is small because the error along the true boresight of the effective unit vector measurement converted from the focal-plane measurement is much smaller than the errors along the other two directions (the first order approximation of $c_{i}$ is zero). For numerical purposes, however, $c_{i}$ may be chosen to be

$$
c_{i}=\frac{1}{2} \operatorname{trace}\left(R_{i}^{\mathrm{NEW}}\right)
$$

where trace denotes the trace of a matrix. That is, $c_{i}$ is the average of the nonzero eigenvalues of $R_{i}^{\mathrm{NEW}}$. Note that using Eq. (30) on $R_{i}^{\mathrm{QUEST}}$ instead of $R_{i}^{\mathrm{NEW}}$ gives $c_{i}=\sigma^{2}$, which yields Eq. (20).

2. The parameter $c_{i}$ will change the property of the measurement covariance matrix, but not that of $H_{i}^{T}\left(\mathcal{R}_{i}^{\mathrm{NEW}}\right)^{-1} H_{i}$. If $H_{i}^{T}\left(\mathcal{R}_{i}^{\mathrm{NEW}}\right)^{-1} H_{i}$ is ill-conditioned, the approach does not help.

3. Since $H_{i}^{T}\left(\mathcal{R}_{i}^{\mathrm{NEW}}\right)^{-1} H_{i}$ remains unchanged with the modified measurement covariance matrix, there is no information loss or gain in the EKF.

4. The approach overcomes the singularity problem based on the very special structure of $H_{i}$ and $R_{i}^{\mathrm{NEW}}: H_{i}^{T} \mathbf{b}_{i}=\mathbf{0}$ and $R_{i}^{\mathrm{NEW}} \mathbf{b}_{i}=\mathbf{0}$. However, if the system does not have such a special structure, the innovation matrix in the EKF will not be singular and the problem no longer exists. 

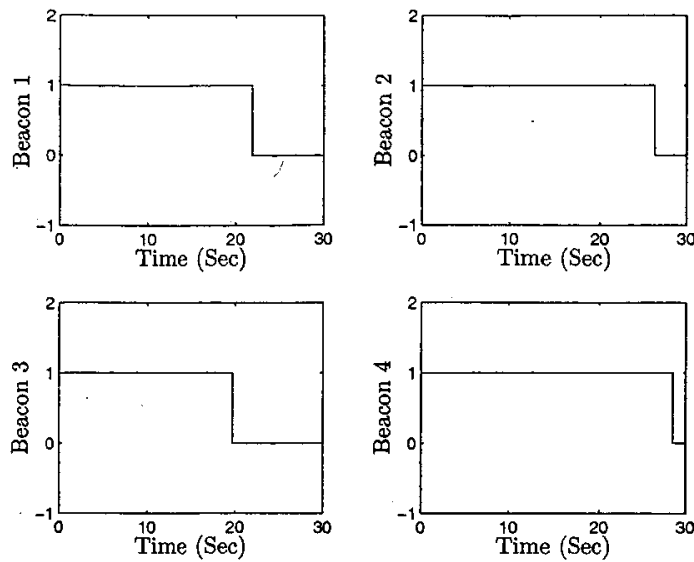

(i) Availability of First Four Beacons

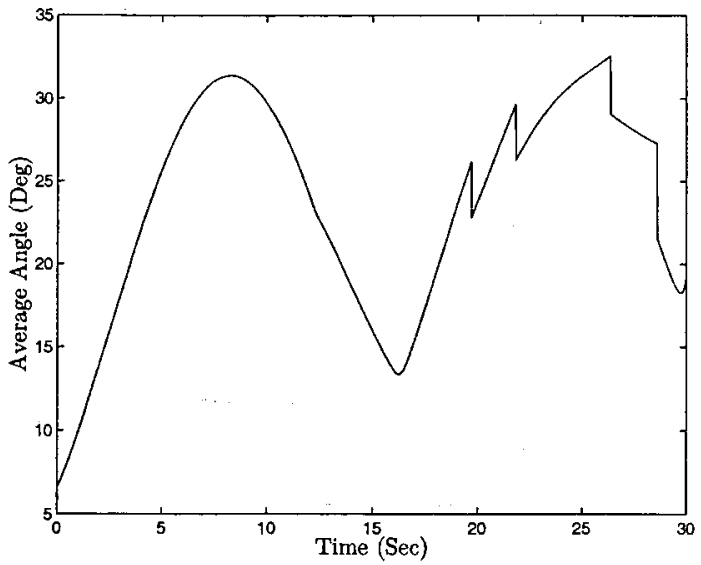

(ii) Average Angle from Boresight

Figure 3 Availability of Beacons and Average Angle from Boresight

\section{SIMULATION RESULTS}

In this section the simulation results using synthetic VISNAV measurements are presented. The VISNAV sensor is only used here to determine attitude for the simulation results. As previously stated, position can also be determined by augmenting the EKF state vector. Eight beacons are used to create the synthetic measurements. The beacon locations in meters are given by

$$
\begin{array}{ccc}
X_{1}=5, \quad Y_{1}=5, & Z_{1}=0 \\
X_{2}=5, \quad Y_{2}=-5, & Z_{2}=0 \\
X_{3}=-5, \quad Y_{3}=5, & Z_{3}=0 \\
X_{4}=-5, \quad Y_{4}=-5, & Z_{4}=0 \\
X_{5}=0.2, \quad Y_{5}=0.2, & Z_{5}=0 \\
X_{6}=0.2, \quad Y_{6}=-0.2, & Z_{6}=0 \\
X_{7}=-0.2, \quad Y_{7}=0.2, & Z_{7}=0 \\
X_{8}=-0.2, \quad Y_{8}=-0.2, & Z_{8}=0
\end{array}
$$

The first four beacons are spread out further than the last four beacons. A 30 second simulation is used with a sampling rate of $100 \mathrm{~Hz}$ for both the VISNAV and gyro measurements. A docking-type motion with a rotation is used in the simulation. The linear motions for both the $x$ and $y$ axes are zero for the entire simulation run. The vehicle performs a linear motion maneuver along the $z$ axis starting 30 meters away at the initial time and ending at 0 at the final time. The initial attitude is given by the identity matrix and the angular velocity is given by $\boldsymbol{\omega}=\left[\begin{array}{lll}0.1 \sin (t) & 0.1 \cos (t) & (3 \times 360 / 30) \times(\pi / 180)\end{array}\right]^{T} \mathrm{rad} / \mathrm{sec}$. The VISNAV sensor FOV is assumed to be 100 degrees. Therefore, any LOS measurement that is greater than 50 degrees from the boresight is not available. Since the last four beacons are "close in," they are available throughout the entire simulation. The availability of the first four beacons is shown in Figure 3(i), where 1 indicates that the beacon is available and 0 indicates that it is not available. The first four beacons are all available until about 20 seconds into the simulation run. Synthetic measurements are generated using the covariance 

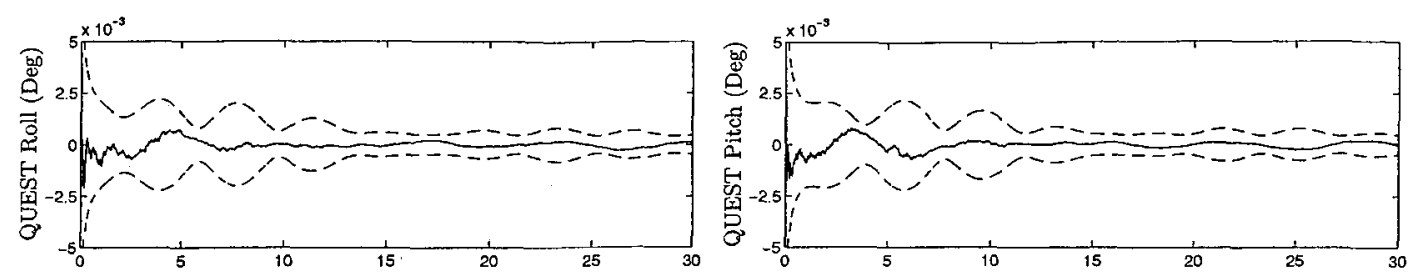

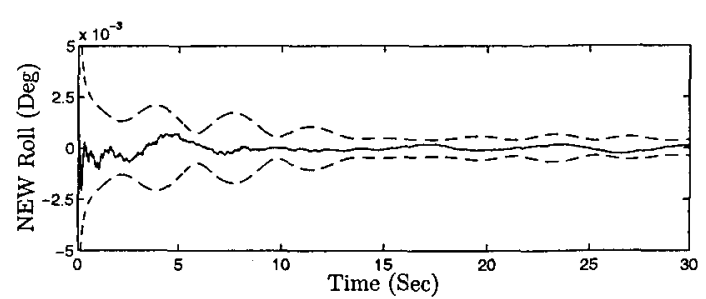

(i) Roll Errors and $3 \sigma$ Bounds
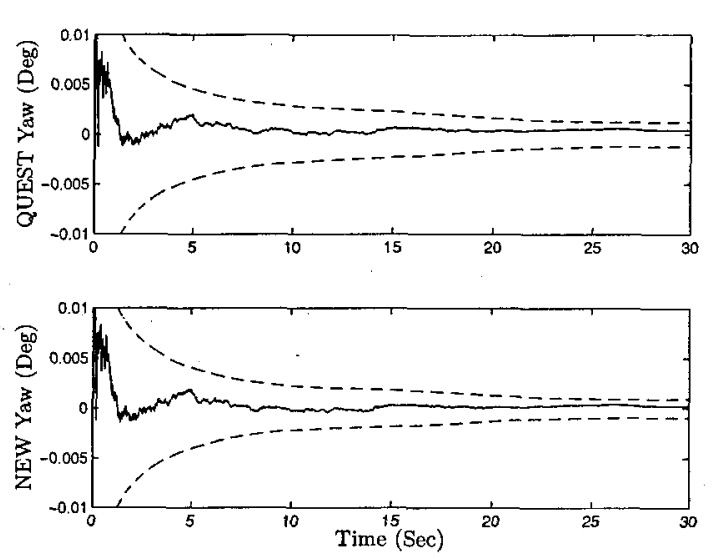

(iii) Yaw Errors and $3 \sigma$ Bounds

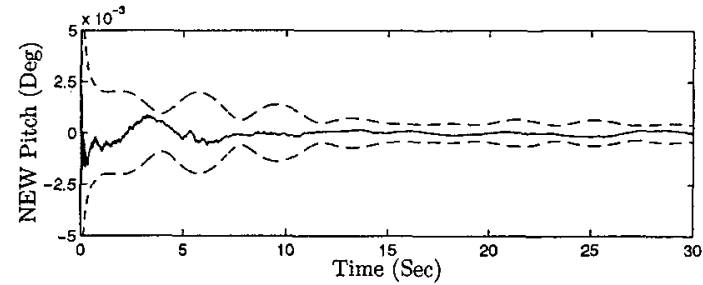

(ii) Pitch Errors and $3 \sigma$ Bounds

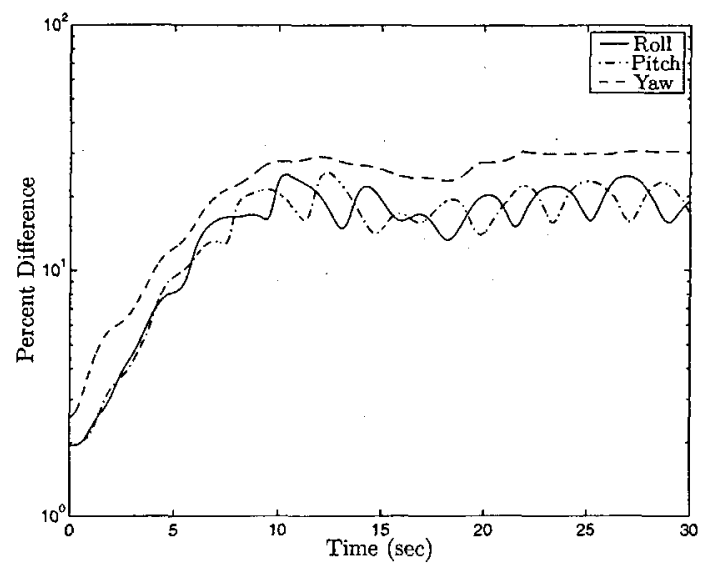

(iv) Percent Difference Between QUEST and NEW Model

Figure 4 Extended Kalman Filter Results

in Eq. (3) with $d=1$ and $\sigma=(100 / 5000) \times(\pi / 180)$ radians. These measurements are then converted into unit-vector form to be used in the EKF. The gyro noise parameters are given by $\sigma_{u}=\sqrt{10} \times 10^{-10} \mathrm{rad} / \mathrm{sec}^{3 / 2}$ and $\sigma_{v}=\sqrt{10} \times 10^{-7} \mathrm{rad} / \mathrm{sec}^{1 / 2}$. See Ref. [17] for a detailed explanation of the gyro model. The initial biases for each axis of the gyro are given by $0.1 \mathrm{deg} / \mathrm{hr}$.

Two EKFs are executed. Both EKFs use the same exact synthetic LOS and gyro measurements, and both use Murrell's version shown in Figure 2. The first EKF uses the QUEST model approach for the measurement covariance with $R_{i}=\sigma^{2} I_{3 \times 3}$. The second EKF uses the new measurement covariance model $R_{i}^{\mathrm{NEW}}$. A plot of the average angle from the boresight for all the available beacons is shown in Figure 3(ii). The discontinuities are due to a loss of a beacon at that specific time. In general we expect that when the average angle is small, then using the QUEST model in the EKF should yield approximately the same solution as using the new model. Plots of the roll, pitch and yaw errors for a typical simulation run, with their respective $3 \sigma$ bounds computed from the error covariance, are 


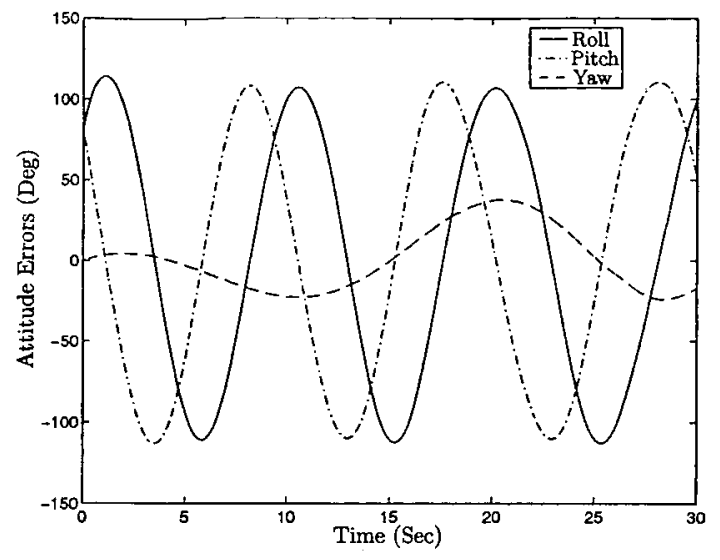

(i) Decomposition Approach

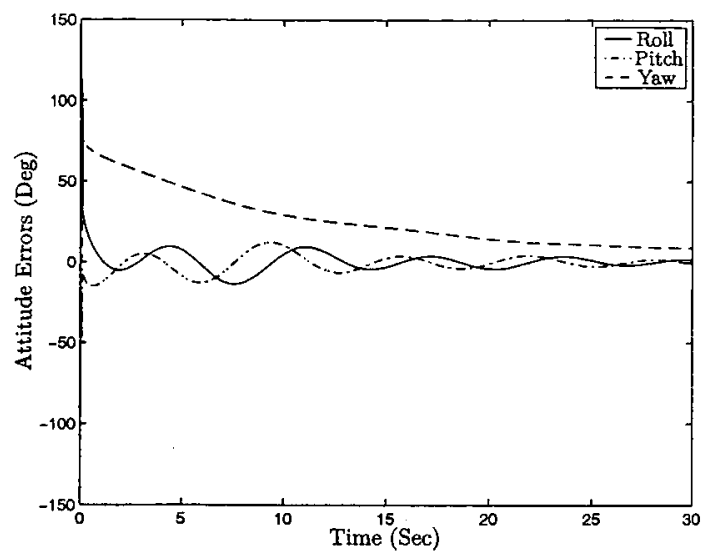

(ii) Rank-One Update Approach

Figure 5 Extended Kalman Filter Results for Large Roll Initial Condition Error

shown in Figures 4(i)-4(iii), respectively. The top plot of each figure corresponds to using the QUEST model in the EKF and the bottom plot corresponds to using the new model approach. At first glance it seems as though both approaches yield nearly identical results, but closer inspection shows that this is not true. To investigate the performance aspects of both filters, the percent difference between the $3 \sigma$ attitude bounds is computed. The results are shown in Figure 4(iv). Using the QUEST model approach gives a $3 \sigma$ bound that is always larger than the new model approach. As expected, when the average angle from the boresight is small the percent differences are negligible. But when the angle is large, using the QUEST model can lead to significantly large errors, as seen by the 30 percent difference in the yaw error. An augmented filter that estimates attitude as well as position has also been developed. The state vector is augmented by six states, which model the three accelerations as zero-mean Gaussian white-noise processes. The position improvements using the new covariance are nearly the same as the attitude improvements, and thus are not shown here for brevity. Overall, the simulation results clearly show that the new covariance model can provide better results over the QUEST model for large FOV sensors.

A comparison between the covariance decomposition approach and the rank-one update approach has also been done. The decomposition approach is found to be sensitive to numerical ill-conditioning effects. In fact large errors are present for the simulation results shown in Figure 4(iv) when the $U-D$ factorization is not used, i.e. running the standard EKF equations. This does not occur with the rank-one update approach. The convergence properties of each approach has also been studied. A roll error of 180 degrees is introduced at the initial condition and the variance for that part of the initial covariance of the EKF is set to $[(220 / 3) \times(\pi / 180)]^{2} \operatorname{rad}^{2}$ for both approaches. For this error both approaches do not converge well. But, for this test the decomposition approach uses the $U-D$ factorization, while the rank-one update approach uses the standard EKF equations. A $U-D$ factorization for the rank-one update approach has also been designed. The results of the $U-D$ factorization EKF version for both the decomposition and rank-one update approaches are shown in Figure 5. Clearly, the rank-one update approach produces better convergence behaviors than the decomposition approach. Several other initial condition errors have also 
been tested, as well as a number of Monte Carlo-type simulations. When using the $U-D$ factorization EKF version for both approaches, the rank-one update approach always shows better convergence behaviors than the decomposition approach. Further improvements may be obtained by using an Unscented filter [18].

\section{CONCLUSIONS}

In this paper a new measurement covariance model was derived that can be used for large field-of-view sensors. The new model was derived using a first-order Taylor series expansion approach of the line-of-sight measurement model. Due to the singular nature of the new covariance, straightforward implementation of the extended Kalman filter was not possible. This was overcome by using one of two approaches. The first approach involves decomposing the covariance matrix into its eigenvalues and eigenvectors, and then performing a transformation of state so that a reduced nonsingular matrix is used in the extended Kalman filter. The second approach adds a simple term, i.e. a rank-one update, to the covariance matrix that does not alter the overall Kalman gain. Simulation results indicate that the new model can yield better estimation results over using the small field-of-view QUEST measurement model in the filter design. Also, a comparison of the two approaches showed that the rank-one update approach can provide better convergence properties than the decomposition approach for large initial condition errors. Also, the rank-one update approach allows a designer to easily implement the extended Kalman filter, even if the QUEST measurement model is used for the measurement covariance. For these reasons, the rank-one update approach is preferred over the decomposition approach.

\section{ACKNOWLEDGEMENT}

The authors wish to thank John L. Junkins from Texas A\&M University for numerous discussions on the VISNAV sensor.

\section{REFERENCES}

1. J. R. Wertz, Mission Geometry; Orbit and Constellation Design and Management, chap. 3, Microcosm Press, El Segundo, CA and Kluwer Academic Publishers, Dordrecht, Netherlands, 2001, pp. 152-167.

2. M. L. Psiaki, F. Martel, and P. K. Pal, "Three-Axis Attitude Determination via Kalman Filtering of Magnetometer Data," Journal of Guidance, Control, and Dynamics, Vol. 13, No. 3, May-June 1990, pp. 506-514.

3. M. Challa, G. Natanson, and C. Wheeler, "Simultaneous Determination of Spacecraft Attitude and Rates Using Only a Magnetometer," AIAA/AAS Astrodynamics Conference, San Diego, CA, July 1996, AIAA-1996-3630.

4. G. Ju and J. L. Junkins, "Overview of Star Tracker Technology and its Trends in Research and Development," Advances in the Astronautical Sciences, The John L. Junkins Astrodynamics Symposium, Vol. 115, 2003, pp. 461-478, AAS-03-285.

5. M. D. Shuster, "Maximum Likelihood Estimation of Spacecraft Attitude," The Journal of the Astronautical Sciences, Vol. 37, No. 1, Jan.-March 1989, pp. 79-88. 
6. D. L. Light, "Satellite Photogrammetry," Manual of Photogrammetry, edited by C. C. Slama, chap. 17, American Society of Photogrammetry, Falls Church, VA, 4th ed., 1980.

7. D. T. Griffith, P. Singla, and J. L. Junkins, "Autonomous On-orbit Calibration Approaches for Star Tracker Cameras," AAS/AIAA Space Flight Mechanics Meeting, San Antonio, TX, Jan. 2002, AAS-02-102.

8. M. D. Shuster, "Kalman Filtering of Spacecraft Attitude and the QUEST Model," The Journal of the Astronautical Sciences, Vol. 38, No. 3, July-Sept. 1990, pp. 377-393.

9. M. D. Shuster and S. D. Oh, "Three-Axis Attitude Determination from Vector Observations," Journal of Guidance and Control, Vol. 4, No. 1, Jan.-Feb. 1981, pp. 70-77.

10. Y. Cheng and J. L. Crassidis, "Particle Filtering for Sequential Spacecraft Attitude' Estimation," AIAA Guidance, Navigation, and Control Conference, Providence, RI, Aug. 2004, AIAA-04-5337.

11. J. L. Junkins, D. C. Hughes, K. P. Wazni, and V. Pariyapong, "Vision-Based Navigation for Rendezvous, Docking and Proximity Operations," 22nd Annual AAS Guidance and Control Conference, Breckenridge, CO, Feb. 1999, AAS-99-021.

12. M. D. Shuster, "Erratum: Kalman Filtering of Spacecraft Attitude and the QUEST Model," The Journal of the Astronautical Sciences, Vol. 51, No. 3, July-Sept. 2003, pp. 359.

13. E. J. Lefferts, F. L. Markley, and M. D. Shuster, "Kalman Filtering for Spacecraft Attitude Estimation," Journal of Guidance, Control, and Dynamics, Vol. 5, No. 5, Sept.-Oct. 1982, pp. 417-429.

14. J. L. Crassidis and J. L. Junkins, Optimal Estimation of Dynamic Systems, chap. 5, Chapman \& Hall/CRC, Boca Raton, FL, 2004.

15. J. W. Murrell, "Precision Attitude Determination for Multimission Spacecraft," Proceedings of the AIAA Guidance, Navigation, and Control Conference, Palo Alto, CA, Aug. 1978, pp. 70-87.

16. J. H. Wilkinson, The Algebraic Eigenvalue Problem, Clarendon Press, Oxford, England, 1965 , pp. 94-97.

17. R. L. Farrenkopf, "Analytic Steady-State Accuracy Solutions for Two Common Spacecraft Attitude Estimators," Journal of Guidance and Control, Vol. 1, No. 4, July-Aug. 1978, pp. 282-284.

18. J. L. Crassidis and F. L. Markley, "Unscented Filtering for Spacecraft Attitude Estimation," Journal of Guidance, Control, and Dynamics, Vol. 26, No. 4, July-Aug. 2003, pp. $536-542$. 